\title{
Neuron-Specific Gene 2 (NSG2) Encodes an AMPA Receptor Interacting Protein That Modulates Excitatory Neurotransmission
}

\author{
Praveen Chander, ${ }^{1}$ (D) Matthew J. Kennedy, ${ }^{2}{ }^{\text {B }}$ Bettina Winckler, ${ }^{3}$ and ${ }^{\mathbb{D}}$ Jason P. Weick ${ }^{1}$
}

https://doi.org/10.1523/ENEURO.0292-18.2018

${ }^{1}$ Department of Neurosciences, University of New Mexico-Health Science Center, Albuquerque, NM 87131,

${ }^{2}$ Department of Pharmacology, University of Colorado-Denver, Aurora, CO 80045, and ${ }^{3}$ Department of Cell Biology, University of Virginia, Charlottesville, VA 22908

\begin{abstract}
Neurons have evolved a number of unique protein-coding genes that regulate trafficking of protein complexes within small organelles throughout dendrites and axons. Neuron-specific gene 2 (NSG2) encodes for one of the most abundant proteins in the nervous system during perinatal development. NSG2 belongs to a family of small neuronal endosomal proteins but its function has remained uncharacterized to date. Here, we show that NSG2 is found in discrete punctae restricted to the somatodendritic arbors of developing mouse and human neurons, and a significant proportion of NSG2 punctae colocalize with postsynaptic HOMER1 and surface-expressed AMPA-type glutamate receptors (AMPARs) at excitatory synapses. Immunoprecipitation revealed that NSG2 physically interacts with both the GluA1 and GluA2 AMPAR subunits in mouse brain. Knock-out of NSG2 in mouse hippocampal neurons selectively impaired the frequency of miniature EPSCs (mEPSCs) and caused alterations in PSD95 expression at postsynaptic densities (PSDs). In contrast, NSG2 overexpression caused a significant increase in the amplitude of mEPSCs as well as GluA2 surface expression. Thus, NSG2 functions as an AMPAR-binding protein that is required for normal synapse formation and/or maintenance, and has unique functions compared with other NSG family members.
\end{abstract}

Key words: AMPAR; endosome; HMP19; human; synaptogenesis; trafficking

\section{Significance Statement}

Because of their morphologic and functional complexity, neurons have evolved specialized proteins like those of the neuron-specific gene family [neuron-specific gene (NSG)1-NSG3]. Important developmental and synaptic functions have been attributed to NSG1/NEEP21 and NSG3/Calcyon, while the function of NSG2/HMP19/NVTA2 has remained uncharacterized. Here, we show that NSG2 localizes to a large proportion of excitatory synapses, interacts with AMPA-type glutamate receptors (AMPARs), and modulates their surface expression during synaptogenesis. Alterations of NSG2 expression affected both the amplitude and frequency of excitatory neurotransmission that is not compensated for by other NSG family members. Thus, NSG2 appears critical for excitatory synapse formation and/or maintenance and forced expression can promote increased synaptic efficacy.

Received July 25, 2018; accepted December 17, 2018; First published January 4, 2019.

The authors declare no competing financial interests.

Author contributions: P.C. and J.P.W. designed research; P.C. and J.P.W. performed research; M.J.K. and B.W. contributed unpublished reagents/analytic tools; P.C. and J.P.W. analyzed data; P.C., M.J.K., B.W., and J.P.W. wrote the paper.
This work was partially supported by grants from NIGMS (GM109089), NSF (NSF1632881), and NINDS (R21NS093442).

Acknowledgements: We thank the University of New Mexico and Cancer Center Fluorescence Microscopy Shared Resource and Flow cytometry core for assistance with confocal image acquisition and analysis and cell sorting. 


\section{Introduction}

Neuron-specific gene 2 (NSG2, neuronal vesicle trafficking-associated 2) belongs to the "neuron-specific gene" family of small, single-pass transmembrane proteins that localize to vesicular compartments within neuronal dendrites. The other two NSG family members are NSG1/NEEP21 and NSG3/Calcyon (Muthusamy et al., 2009; Norstrom et al., 2010; Lasiecka et al., 2014), which have been shown to have a number of critical protein trafficking functions primarily involved with neural development and synaptic plasticity. Interestingly, Human NSG2 shares only 50\% sequence homology with NSG1 and a $30 \%$ sequence homology with NSG3 at the amino acid level (Muthusamy et al., 2009). NSG2 is one of the most highly expressed transcripts and proteins during human neural differentiation and synapse formation (Kang et al., 2011; Miller et al., 2014; Barford et al., 2017; Murillo et al., 2017). It is expressed widely throughout the brain (Sabéran-Djoneidi et al., 1995) with significant overlapping expression with NSG1 but some degree of divergence with NSG1 (Barford et al., 2017). At the cellular level, NSG2 is found extensively localized to the transGolgi network as well as in dendritic endosomes, and was recently found to be expressed transiently at the cell surface (Yap et al., 2017). Interestingly, NSG2 also contains a Secretogranin III domain as well as an atypical EGF-like motif (Sabéran-Djoneidi et al., 1995), making it a good candidate for regulating signaling and exocytosis. However, while much is known about the spatial distribution of NSG2 in dendritic endosomes, its role in neuronal function has remained elusive.

One major function attributed to both NSG1 and NSG3 is the regulated trafficking of the AMPA-type glutamate receptors (AMPARs) at postsynaptic densities (PSDs), (for review, see Yap and Winckler, 2012; Muthusamy et al., 2015). For instance, NSG3 is found in RAB5 ${ }^{+} / E E A 1^{+}$early endosomes, interacts with AP2 and AP3, and is critical for clathrin-mediated endocytosis of AMPARs (Xiao et al., 2006; Muthusamy et al., 2012, 2015). Knock-out of NSG3 impairs long-term depression (LTD)-mediated AMPAR endocytosis, while overexpression reduces GluA1 and GluA2 surface expression and impairs performance on a fear extinction paradigm (Xiao et al., 2006; Kruusmägi et al., 2007; Davidson et al., 2009; Vazdarjanova et al., 2011). NSG1, on the other hand, is colocalized to a limited extent with the early endosome marker RAB5 and the recycling endosome marker RAB11 (Steiner et al., 2002; Yap et al., 2017). Overexpression of the C-terminal tail of NSG1 acts as a dominant negative and prevents recycling of GluA1/2-containing AMPARs following NMDA-mediated internalization (Alberi et al., 2005; Steiner et al., 2005).

\footnotetext{
We also thanks Fernando Valenzuela and Chan Choo Yap for helpful discussions regarding experimental design and methodology.

Correspondence should be addressed to Jason P. Weick at jpweick@ salud.unm.edu.

https://doi.org/10.1523/ENEURO.0292-18.2018

Copyright (C) 2019 Chander et al.

This is an open-access article distributed under the terms of the Creative Commons Attribution 4.0 International license, which permits unrestricted use, distribution and reproduction in any medium provided that the original work is properly attributed.
}

Together these proteins play complementary roles in regulating internalization (NSG3) and recycling (NSG1) to promote AMPAR sorting between intracellular compartments and the plasma membrane during synaptic plasticity at established synapses. However, the predominant expression of NSG2 during early periods of development (Sabéran-Djoneidi et al., 1995; Barford et al., 2017) led us to consider whether it may function during early periods of synaptogenesis. Further, while much is known about AMPAR trafficking at mature synapses, less is known regarding the mechanisms by which AMPARs are trafficked during synaptogenesis.

Here, we report that NSG2 is a critical regulator of neuronal AMPAR surface expression in hippocampal cultures during early periods of neuronal development. In agreement with previous reports (Yap et al., 2017), we found NSG2 strongly localized to the trans-Golgi network as well as in a punctate pattern selectively in developing $\mathrm{MAP}^{+}$dendrites, but is excluded from axons. In addition, a significant proportion of NSG2 punctae colocalized with synaptic markers including AMPAR subunits. Co-immunoprecipitation (Co-IP) and capillary immunoelectrophoresis experiments demonstrated a physical interaction with both GluA1 and GluA2. Overexpression and CRISPR-mediated knock-out of NSG2 altered AMPAR surface expression as well as AMPAR-mediated postsynaptic currents (PSCs). These data are the first to demonstrate a role for NSG2 in the regulation of AMPAR surface expression and implicate NSG2 as a potential partner of AMPARs in regulated endosomal trafficking in developing dendrites.

\section{Materials and Methods}

\section{Cell culture, transfection, and transduction}

HEK293T cells and Neuro-2a cells were maintained in DMEM supplemented with $10 \%$ fetal bovine serum (FBS) and $1 \times$ penicillin/streptomycin (Pen/Strep; Thermo Fisher Scientific). Cells at $90 \%$ confluence were passaged every 3 d using 0.05\% trypsin (Thermo Fisher Scientific). HEK293T cells were transfected using the calcium phosphate method with $10 \mu \mathrm{g}$ plasmid DNA. Neuro-2a cells were transfected using Lipfectamine 3000 (Thermo Fisher Scientific) as per manufacturer's recommendation. Hippocampal pyramidal neurons were derived from postnatal; P0-P1 C57Bl/6J pups (The Jackson Laboratory) of either sex. Briefly, brains were isolated, and the hippocampus was dissected in ice-cold Hanks' balanced salt solution (HBSS) solution (Sigma) supplemented with $20 \% \mathrm{FBS}$ and $\mathrm{NaHCO}_{3}$ (4.2 mM), HEPES (1 mM; Sigma); $\mathrm{pH}$ 7.4. Dissected hippocampi were digested for $10 \mathrm{~min}$ with $0.25 \%$ trypsin (Thermo Fisher Scientific). Tissue was washed and dissociated using fire polished Pasteur pipettes of decreasing diameter in ice cold HBSS containing DNase (1500 U; Sigma). The cells were pelleted, resuspended in plating media and plated at a density of 4-5 $\times 10^{5}$ cells/12-mm coverslip (Electron Microscopy Sciences) coated with poly-Ornithine $(0.1 \mathrm{mg} / \mathrm{ml}$; Sigma, catalog \#4638) and laminin (5 $\mathrm{g} / \mathrm{ml}$; Thermo Fisher Scientific). Cells were allowed to adhere for $20 \mathrm{~min}$ before addition of $0.5 \mathrm{ml}$ of plating media containing Neurobasal supplemented with $1 \times \mathrm{B} 27,2 \mathrm{mM}$ Glutamax, $0.5 \mathrm{mg} / \mathrm{ml}$ 
Pen/Strep and 5\% FBS (all from Thermo Fisher Scientific) for the first $24 \mathrm{~h}$. Serum was eliminated from this media after $24 \mathrm{~h}$ and again replaced after $48 \mathrm{~h}$ supplemented with $4 \mu \mathrm{M}$ cytosine 1- $\beta$-D-arabinofuranoside (Ara-C; Sigma). Neurons were fed by replacing half the volume of spent media with fresh media without serum or Ara-C every week. All animal procedures were performed in accordance with University of New Mexico-Health Science Center animal care committee's regulations. Neurons on coverslips were transduced with lentivirus Multiplicity Of Infection (MOI 2-3) on day 4 in culture for CRISPR studies or day 7 in culture for NSG2 overexpression studies and assayed at the days indicated in results. Human pluripotent stem cells (hPSC; line WA09) were maintained in mTesR (STEMCELL Technologies) and passaged using $1 \mathrm{U} / \mathrm{ml}$ dispase (STEMCELL Technologies) as per manufacturer's recommendation once the cells reached $80 \%$ confluence. hPSC-derived neurons (hPSNs) were differentiated from one six well plate of hPSCs. Briefly, the cells were lifted using dispase and allowed to grow in suspension in a T75 flask for $4 \mathrm{~d}$ in mTeSR. From day 5 to day 20 , cells were grown in neural induction media (DMEM/F12; Sigma) supplemented with $1 \times$ concentrations of N2, Glutamax, and Pen/Strep (Thermo Fisher Scientific) and $2 \mu \mathrm{g} / \mathrm{ml}$ heparin (Sigma) to derive neurospheres with $2 / 3$ rd volume of media replaced every other day. On day 21, 8-10 neurospheres were plated down/ 12 -mm glass coverslip in a 24-well dish-coated with polyornithine and laminin as described above and allowed to attach for a minimum of $4 \mathrm{~h}$ before feeding with $0.5 \mathrm{ml}$ neural differentiation media [50:50 mixture of Neurobasal: DMEM/F12 supplemented with $1 \times$ concentrations of N2, Glutamax, Pen/Strep, and $10 \mathrm{ng} / \mathrm{ml}$ of cAMP (Sigma), BDNF and GDNF (Peprotech), and $100 \mathrm{mM}$ ascorbic acid (Sigma)]. Half volume of media was replaced every other day until the assayed days mentioned in the Results section.

\section{Plasmids and lentivirus production}

Episomal vectors encoding mCherry or NSG2-mCherry (NSG2-mC) were generated in house by cloning either mCherry or the open reading frame of human NSG2 (NM_015980.4) in frame with mCherry into the pCS2+ mammalian expression plasmid (kind gift from Bill Bement, University of Wisconsin). The NSG1-GFP plasmid was a kind gift from Bettina Winckler (University of Virginia). The pSIN REP5-GFP-GluA2(R) was purchased from Addgene (Addgene, catalog \#24005) and the YFPGluA1 was previously described (Sharma et al., 2006). Lentiviral constructs were generated by cloning either mCherry or NSG2-mC downstream of the Synapsin-1 promoter in the pFCK plasmid (Dittgen et al., 2004). CRISPR/Cas9 guide RNAs (gRNAs) targeting mouse NSG2 (NM_008741.4) were generated using the CRSPR design webtool (https://zlab.bio/guide-design-resources). gRNAs (MsNSG2\#1: 5'- GCGTGATGAGAGGGACGGTC$3^{\prime}$ and MsNSG2\#2: 5'-CGTCCCTCTCATCACGCCCT$3^{\prime}$ ) were cloned into pL-Crispr.EFS.GFP (Addgene, catalog \#57818) into the BsmBI site as previously suggested (Heckl et al., 2014). Lentivirus were produced in HEK293T cells using calcium phosphate transfection of a $15 \mu \mathrm{g}$ total mixture of lentiviral DNA and packaging plasmids psPax2 and pMD2.G (gifts from Didier Trono, Addgene, catalog \#12260 and catalog \#12259, respectively) at a ratio of 3:2:1 for lentivirus production; 36-48 h posttransfection, lentivirus-containing media were harvested and concentrated using Lenti- $X$ concentrator (Clontech) as per manufacturer's recommendations. Pellets were resuspended in ice cold DMEM, aliquoted, and frozen at $-80^{\circ} \mathrm{C}$ till use.

\section{Cell and whole-brain lysates}

HEK293T cells were lysed $48 \mathrm{~h}$ post-transfection by incubating cells for $30 \mathrm{~min}$ in ice-cold lysis buffer containing: $50 \mathrm{mM}$ Tris base, $150 \mathrm{mM} \mathrm{NaCl}, 1 \mathrm{mM}$ EDTA, $1 \%$ Triton X-100, and $1 \times$ protease inhibitor cocktail (Thermo Fisher Scientific); $\mathrm{pH}$ 7.4. Whole-brain homogenate was prepared by harvesting mouse brains from P0 pups and subjecting them to a brief ice-cold PBS wash. Brains were then homogenized by sonication $(5 \times 10 \mathrm{~s})$ in ice cold lysis buffer using a sonic dismembrator (Fisher Scientific, model F60) with output power set at 1. Protein amounts in the supernatant was quantified using the BCA assay (Thermo Fisher Scientific) and was aliquoted and frozen at $-80^{\circ} \mathrm{C}$ until use after cold centrifugation at $10,000 \times g$ for $15 \mathrm{~min}$.

\section{Co-IP}

For AMPAR Co-IP $2 \mathrm{mg}$ total protein was incubated with $4 \mu \mathrm{g}$ of either mouse anti-GluA1 (Clone RH95; Millipore), mouse anti-GluA2 (Clone 6C4; Millipore), or control mouse IgG1 (Clone G3A1; Cell Signaling Technology) antibodies overnight at $4^{\circ} \mathrm{C}$, with gentle rocking. Immune complexes were precipitated for $2 \mathrm{~h}$ at $4^{\circ} \mathrm{C}$ using $20 \mu \mathrm{l}$ protein $A / G$ agarose beads (Santa Cruz Biotechnology). For NSG2-mC Co-IP, $1.5 \mathrm{mg}$ total protein was incubated with either $50 \mu \mathrm{l}$ anti-RFP mAb-agarose (MBL International Corporation) or RFP-Trap_M (ChromoTek Inc.) according to manufacturer's instructions. Agarose beads from the above reactions were washed and denatured in $50 \mu \mathrm{l} 1 \%$ SDS, and $20 \mu \mathrm{l}$ was loaded on an SDS-PAGE gel for Western blotting or $2 \mu \mathrm{l}$ of the supernatant was subject to capillary electrophoresis.

\section{Western blotting and capillary electrophoresis}

SDS denatured proteins were processed via SDSPAGE and transferred to FL-PVDF membranes (Li-COR) for traditional immunoblotting. For Western blotting primary antibodies used were rabbit anti-NSG2 (1:500; Abcam), mouse anti- $\beta$-actin (1:5000; Clone AC-15; Thermo Fisher Scientific), and mouse anti-GAPDH (1:500; Clone 6C5; Thermo Fisher Scientific). Secondary antibodies used for detection of primary antibodies were goat antimouse $800 \mathrm{CW}(1: 15,000 ; \mathrm{Li}-\mathrm{COR})$ and goat anti-rabbit 680RD (1:15,000; Li-COR), and blots were scanned using the Odyssey infrared imager and acquired on the Image Studio Lite software suite (version 3.1, Li-COR). Capillary electrophoresis was performed on the fully automated Wes system (ProteinSimple) following the manufacturer's recommendations. Briefly, $0.5 \mu \mathrm{g}$ protein lysate (Input lanes) or $2 \mu$ l protein (IP lanes) were mixed with $2 \mu$ l of the 
$5 \times$ Master mix containing SDS and DTT. The samples and protein standard were boiled at $95^{\circ} \mathrm{C}$ for $5 \mathrm{~min}$. The samples were dispensed into microplates containing blocking buffer, primary and secondary antibody and wash buffer in independent wells for sequential processing. The plate was briefly spun and loaded into the instrument for electrophoretic separation of proteins in capillary tubes containing a $12-$ to $250-k D a$ separation matrix. The chemiluminescence based electrophoretogram was autogenerated and digitally-rendered bands were generated from the chemiluminescent peaks using the Compass software (ProteinSimple). For the Wes, primary antibodies were rabbit anti-NSG2 (1:300), mouse anti-GluA1 (1:100), and mouse anti-GluA2 (1:50). HRP-conjugated secondary anti-mouse and anti-rabbit antibodies were used at the predefined concentrations provided by the manufacturer.

\section{T7 endonuclease I assay}

Neuro-2a cells were transfected with either the control or CRISPR NSG2 KO gRNA plasmids and allowed to express the constructs for $48-60 \mathrm{~h}$. The cells were then harvested and a purified $\mathrm{GFP}^{+}$cells population was obtained by flow cytometry. The genomic DNA from the $\mathrm{GFP}^{+}$cell population was extracted using a commercially available kit (Zymo Research); $100 \mu \mathrm{g}$ of genomic DNA was used as a template for PCR amplification of a fragment surrounding the putative gRNA cleavage site using the following primer pair: Fwd 5'-TCCCCGGACAATGGGAAT CATG-3' and Rev 5'-GTGGCTGGAAGAATGAAAGGAT-3'. Amplicons were then subjected to a single cycle of denaturation and renaturation to generate heteroduplex molecules containing mismatches which could be recognized and cleaved using the T7 Endonuclease I enzyme (New England Biolabs). The products of the reaction were resolved on a $2 \%$ agarose gel containing $1 \times$ gel red stain (Biotium) and imaged on a gel documentation system (Bio-Rad). The relative band intensities of the cut fragments to the uncut fragment were used to calculate the gRNA-mediated cleavage efficiency.

\section{Immunocytochemistry}

Neurons on coverslips were fixed with $4 \%$ paraformaldehyde/4\% sucrose for $15 \mathrm{~min}$, rinsed three times for 5 min in PBS (Sigma), and permeabilized using 0.2\% Triton X-100 for 10 min (except when staining for surface GluA1 and GluA2). Cells were blocked with $10 \%$ donkey serum in PBS for $1 \mathrm{~h}$, followed by an overnight incubation of primary antibody in $5 \%$ donkey serum at $4{ }^{\circ} \mathrm{C}$. Primary antibodies consisted of rabbit anti-NSG2 (1:500; Abcam), goat anti-NSG1 (1:400; Everest Biotech), mouse antiGFAP (1:1000; Neuromab), chicken anti- $\beta_{\mathrm{II}}$-tubulin (1: 500; Millipore), rabbit anti-Homer1 (1:1000; Synaptic Systems, GmbH), guinea pig anti-Homer1 (1:200; Synaptic Systems), mouse anti-Synapsin-1 (1:2000; Synaptic Systems), chicken anti-MAP2 (1:5000; Biolegend), mouse anti-SMI312 (1:1000; Biolegend), mouse anti-PSD95 (Clone 7E3, 1:100; Thermo Fisher Scientific), and antibodies targeting the N terminus of GluA1 and GluA2 (1:100; see above, Co-IP). Following primary antibody incubation, cells were washed thrice with PBS and incubated for $1 \mathrm{~h}$ with secondary antibody in $5 \%$ donkey serum. Conju- gated secondary antibodies used were: DyLight 488, 550, and 647 (1:1000; Thermo Fisher Scientific), donkey antiguinea pig CF555 and goat anti-chicken CF647 (both at 1:500; Sigma). Cells were washed with PBS and then in some cases treated with DAPI $(1: 10,000$ in PBS; Thermo Fisher Scientific), followed by three washes with $1 \times$ PBS, and mounted on superfrost slides on Fluoromount-G as an anti-quenching reagent (Southern Biotech). In our hands, the GluA1 antibody described above did not yield much success with hPSNs. Therefore, we conducted live staining for surface GluA1 on hPSNs with a custom developed rabbit anti-GluA1 targeting the extracellular epitope of GluA1 (1:200; a kind gift from Dr. Matthew Kennedy, University of Colorado Denver). The cells were incubated live with the antibody for 15 min before three brief PBS washes and fixation with 4\% PFA. The postfixation treatments and secondary antibody incubations were same as described above.

\section{Confocal imaging and analysis}

Confocal z-stacks were acquired on the Zeiss LSM800 airyscan confocal microscope using the 63x/1.40NA Oil objective. Sequential frame acquisition was set to acquire an average of 10 planes per stack at 16 bit and a minimum of $1024 \times 1024$ resolution. Channel gain settings were optimally adjusted to minimize saturation of punctae and were maintained across experimental groups. Unmodified images were used for all analyses and linear scaling was applied on images only for presentation purposes using Zen Black 2.3 (https://www.zeiss.com/microscopy/us/ downloads/zen.html). Fluorescent signal colocalization on the single planes from a stack and quantification of punctae number was performed using the colocalization plugin for ImageJ ComDet version 0.3.4 (https://github.com/ekatrukha/ComDet) as previously described (Esteves da Silva et al., 2015). Punctae integrated fluorescence intensity over area measurements were performed with ImageJ $1.48 \mathrm{v}$ (https://imagej.nih.gov/ij/). For each experimental group an average of $>200 \mu \mathrm{m}$ dendrite was quantified for 8-10 images per experiment and repeated for a total of three biological replicates.

\section{Electrophysiology}

Whole-cell patch-clamp recordings were performed a previously described (Weick et al., 2013) with minor modifications. The extracellular solution was a modified HBSS that contained: $140 \mathrm{mM} \mathrm{NaCl}, 3 \mathrm{mM} \mathrm{KCl}, 2 \mathrm{mM} \mathrm{CaCl}_{2}, 1$ $\mathrm{mM} \mathrm{MgCl} 2,15 \mathrm{mM}$ HEPES, and $23 \mathrm{mM}$ glucose; $\mathrm{pH} 7.4$, $300 \mathrm{mOsm}$. Recording pipettes with resistances of 3-5 $\mathrm{M} \Omega$ were filled with an intracellular recording solution containing the following: $121 \mathrm{mM}$ K-gluconate, $20 \mathrm{mM}$ $\mathrm{KCl}, 2 \mathrm{mM} \mathrm{MgCl}$, $10 \mathrm{mM}$ EGTA, $10 \mathrm{mM}$ HEPES acid, 2 $\mathrm{mM} \mathrm{Mg}{ }^{2+}$-ATP, and $0.2 \mathrm{mM} \mathrm{Na}^{+}$-GTP; pH 7.2, 290 mOsm. Pharmacological antagonists picrotoxin $(50 \mu \mathrm{M}$; Tocris), TTX (1 $\mu \mathrm{M}$; Tocris), and AP5 (25 $\mu \mathrm{M}$; Sigma) were bath applied in the external solution. Each experiment consisted of 10-13 cells per group, and experiments were repeated three times on different cultures at the same time points indicated in the results. Neurons were visualized using an Olympus Optical BX51WI microscope (Olympus Corp.) with differential interference contrast op- 
tics at $40 \times$. Recordings were obtained using a MultiClamp 700B amplifier (Molecular Devices), filtered at 4 $\mathrm{kHz}$ and sampled at $100 \mathrm{kHz}$ using a Digidata 1322A analog-to-digital converter (Molecular Devices). Wholecell capacitance was fully compensated but series resistance was not compensated. Access resistance was monitored before and after recordings, and cells with resistances $>20 \mathrm{M} \Omega$ at either point were discarded from analyses. Miniature EPSCs (mEPSCs) were measured using a holding potential of $-70 \mathrm{mV}$, while outward potassium currents were elicited by a voltage-step protocol from -50 to $+50 \mathrm{mV}$, and all recordings were performed at $32^{\circ} \mathrm{C}$. Step protocols were used to verify the lack of inward sodium current in all cells used for subsequent analysis. Data were stored on a computer hard disk and PSCs were analyzed using MiniAnalysis software (Synaptosoft), while potassium currents were analyzed using Clampfit v. 10.0 (Molecular devices). The cells used for mEPSC recordings for NSG2 knock-out (control GFP and CRISPR NSG2 KO) were injected with Lucifer yellow (10 $\mathrm{mM}$; Thermo Fisher Scientific) included in the internal solution. After recordings cells were immediately fixed and stained for NSG2 as described above (see above, Immunocytochemistry). Only cells showing a lack of NSG2 (indicating NSG2 KO) were included for subsequent analysis.

\section{Statistical analyses}

Student's $t$ tests were used to determine whether mean differences between groups (e.g., control mCherry vs NSG2-mC) were significant and were considered significant a priori if $p<0.05$. The number of samples used for statistical analyses $(n)$ refers to the number of cells assayed per group accumulated from three independent biological replicates for all imaging-based assays and electrophysiology experiments. For analysis of synaptic and NSG2 punctae, $n$ 's are still reported by the criteria above but at least 1500 total punctae were included across cells/replicates. Data are reported as mean \pm SEM.

\section{Data availability}

Data generated and analyzed in this study are available from the corresponding author on request.

\section{Results}

\section{NSG2 is localized to PSDs of excitatory synapses}

NSG2 is one of the most highly upregulated and abundant mRNAs during early neuronal development across multiple species, including human (Kang et al., 2011; Miller et al., 2014; Barford et al., 2017; Murillo et al., 2017; Floruta et al., 2017). To determine whether NSG2 protein was similarly upregulated, we first used differentiation of cortical neurons derived from human pluripotent stem cells (hPSCs) and assayed for NSG2 using an antibody previously shown to detect the rodent protein (Barford et al., 2017). Robust NSG2 expression was observed in $98 \%$ of the $\beta_{I I I}$-tubulin ${ }^{+}$neurons analyzed ( $n=151 / 154$ neurons from five independent experiments) at days 30 and 50 in culture (Extended Data Fig. 1-1A,B). These timepoints correspond to periods before and following the formation of functional synapses in hPSC-derived neurons (hPSNs), respectively. NSG2 was not found in neuroepithelial cells at day 30 that were $\mathrm{PAX6}^{+} / \mathrm{MAP}^{-}$ (Extended Data Fig. 1-1C), suggesting its upregulation following the final mitotic division. Similarly, NSG2 was detected by $4 \mathrm{~d}$ in vitro (DIV4) in cultured mouse hippocampal neurons (Fig. 1A), an early time point before the creation of functional synapses (Grabrucker et al., 2009). NSG2 remained robustly expressed in all hippocampal neurons examined ( $n=391 / 391$; from six independent cultures) at all time points assayed up to DIV30 (Extended Data Fig. 1-2). Similar to previous reports, NSG2 was found robustly aggregated in perinuclear regions consistent with localization to the Golgi apparatus (SabéranDjoneidi et al., 1995) as well as in distinct punctae throughout $\beta_{\mathrm{III}}$-tubulin ${ }^{+}$neurites (Fig. $1 A, B$, yellow box magnified; Extended Data Fig. 1-1, blue, arrows). At DIV4, NSG2 was absent from GFAP ${ }^{+}$astrocytes in regions that did not have overlapping $\beta_{\mathrm{III}}$-tubulin ${ }^{+}$neurites (Fig. 1A, $B$, yellow box magnified, cyan, arrowheads). This was also evident in cultures of human neurons and glia at day 50 (Extended Data Fig. 1-3A), supporting the neuron-specific nature of NSG2 across species. At DIV14 in mouse neurons, the number of NSG2 punctae were dramatically increased as neuronal arbors became more elaborate (Fig. 1C). At this time point, NSG2 punctae were restricted to the somatodendritic compartment as MAP2 ${ }^{+}$dendrites showed robust staining (Fig. $1 C, D$, yellow box magnified; Extended Data Fig. 1-3B, blue, arrows), whereas SMI$312^{+}$axons were devoid of NSG2 (Fig. $1 C, D$, yellow box magnified; Extended Data Fig. 1-3B, cyan, arrowheads). Together, these data support the neuron-specific nature of NSG2 and show its punctate localization restricted to somatodendritic arbors of human and mouse neurons.

In contrast to previous reports suggesting that NSG2 protein is nearly absent in brain following early postnatal development (Sabéran-Djoneidi et al., 1995), Barford et al., recently showed that expression of NSG2 was maintained in cortex up to the period of adolescence (P16) but was absent from cerebellum in adults (P60; Barford et al., 2017). Due to sequence homology between NSG family members we determined whether the antibody used for immunostaining was specific for NSG2. Western blot analysis was used to validate the specificity of the NSG2 antibody, which revealed a band of $\sim 47 \mathrm{kDa}$ in lysates taken from HEK293T cells overexpressing NSG2 (19 kDa) linked to the mCherry (28 kDa) fluorophore (NSG2-mC; Fig. 1E, upper panel, middle lane). However, no bands were detected in lysates taken from HEK293T cells overexpressing NSG1 (21 kDa) linked to GFP (27 $\mathrm{kDa}$; Fig. 1E, upper panel, right lane) or untransfected HEK293T cell lysates (Fig. 1E, upper panel, left lane). $\beta$-Actin served as a loading control (Fig. 1E, lower panel). Further, to determine the expression profile of endogenous NSG2 protein over time, we performed Western blotting of mouse whole-brain lysates. We found that while NSG2 has its greatest expression during the perinatal period and is gradually downregulated thereafter, it 
A
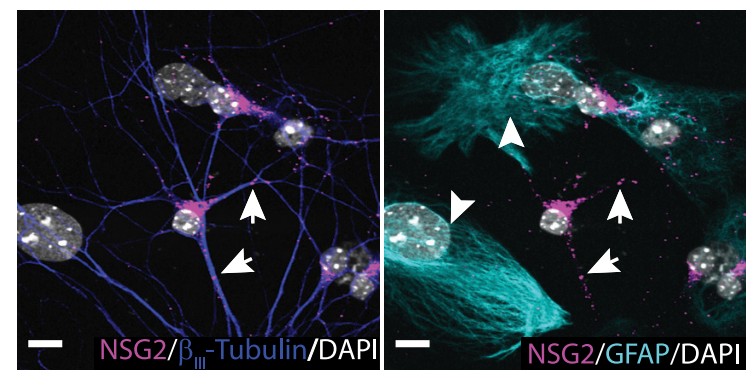

C
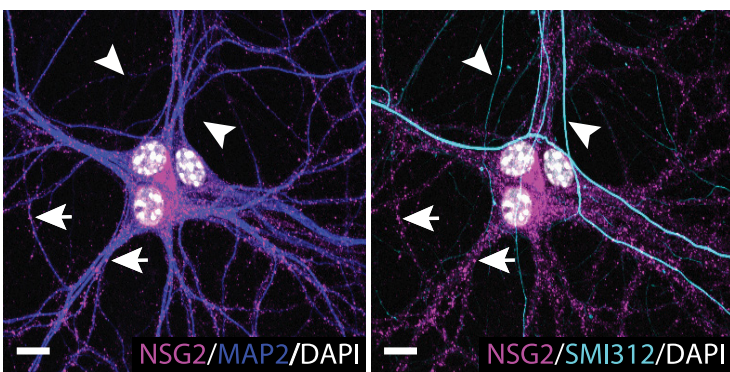

E

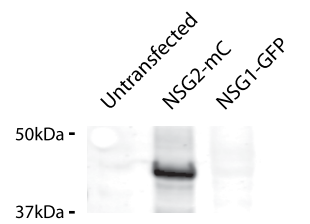

Blot: NSG2

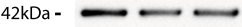

Blot: $\beta$-actin
$\mathbf{F}$

$\begin{array}{lllllll}\text { P0 } & \text { P7 } & \text { P14 } & \text { P21 P30 P60 }\end{array}$

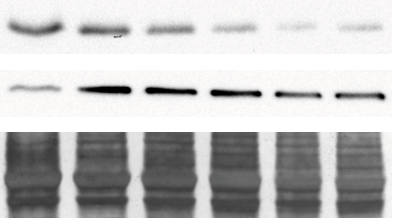

Coomassie loading control
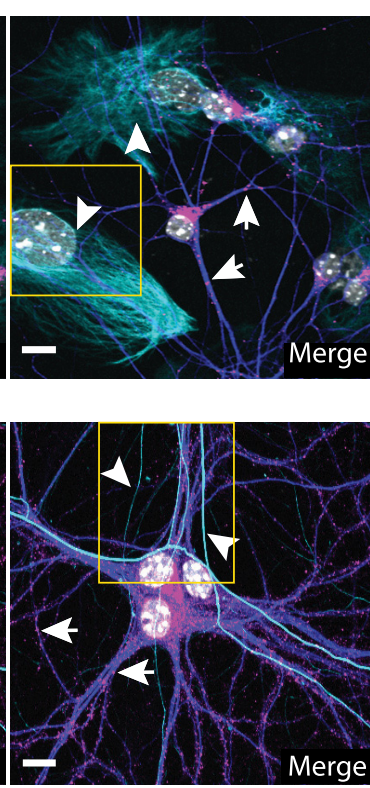

G

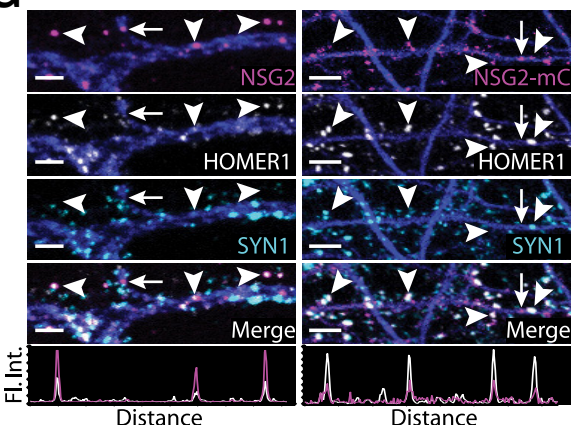

B

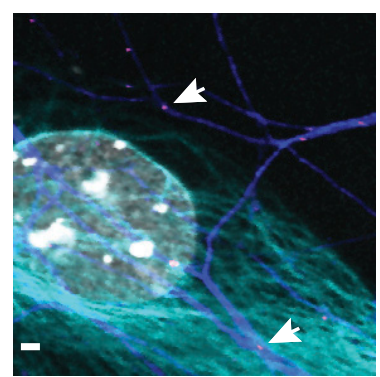

D

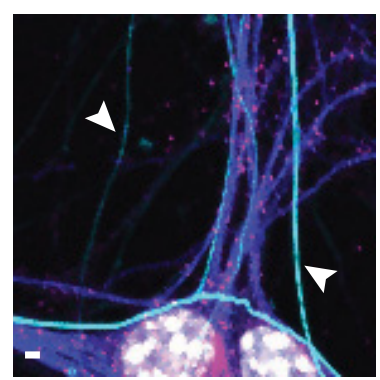

H

Figure 1. A subset of NSG2 punctae localize to PSDs of excitatory synapses. $\boldsymbol{A}$, Representative confocal images of primary hippocampal neurons (DIV4) illustrate a perinuclear as well as punctate NSG2 expression pattern (magenta; arrows) in $\beta_{\text {III }}$-tubulin ${ }^{+}$ neurons (blue). No NSG2 expression was observed in GFAP ${ }^{+}$astrocytes (cyan; arrowheads). The punctate expression pattern of NSG2 was found in all neurons tested up to DIV30 (Extended Data Fig. 1-1). $\boldsymbol{B}$, Magnified image of yellow box in the merge panel of $\boldsymbol{A}$. NSG2 puncta (magenta; arrows) are present only within $\beta_{\mathrm{III}}$-tubulin ${ }^{+}$neurons (blue). $\boldsymbol{C}$, Immunofluorescent images illustrate NSG2 (magenta; arrows) punctae exclusively in $\mathrm{MAP}^{+}$(blue) dendrites and absence of NSG2 punctae in SMI312 ${ }^{+}$axons (cyan; arrowheads) at DIV14. D, Magnified image of yellow box in the merge panel of $\boldsymbol{C}$. $\boldsymbol{E}$, Representative Western blotting image demonstrating the specificity of the NSG2 antibody in detecting NSG2 (NSG2-mC; top panel, 48-kDa band; middle lane) but not the closely-related protein NSG1 (NSG1-GFP; top panel, $\sim 48$ kDa). Bottom panel shows the $\beta$-actin loading control. $\boldsymbol{F}$, Representative Western blotting image demonstrating the expression profile of NSG2 from mouse whole-brain lysate during development (top panel, 19-kDa band). While GAPDH was used as a loading control (middle panel), it shows variability across development. The blot was also stained with Coomassie (bottom panel) to demonstrate equal protein loading. G, Confocal images showing endogenous NSG2 (left panels) and overexpressed NSG2-mC (right panels) colocalized with HOMER1 ${ }^{+}$punctae (arrowheads; HOMER1 panel and merge). Endogenous NSG2 and NSG2-mC were frequently found adjacent to Synapsin1 ${ }^{+}$punctae (e.g., leftmost arrowheads). Individual panels for HOMER1 ${ }^{+}$(white) and SYN1 ${ }^{+}$(cyan) are shown for clarity. The colocalization profile of NSG2 or NSG2-mC (magenta trace) with HOMER1 (white trace) for the indicated punctae are indicated in the bottom panels. Some NSG2 punctae were not found colocalized to either HOMER1 or SYN1 punctae (arrow). $\boldsymbol{H}$, Quantification of colocalization of Homer1 with either endogenous NSG2 or NSG2-mC. Bars represent mean \pm SEM for $n=10$ for each group; $* p=0.024$. Scale bar $=10 \mu \mathrm{m}(\boldsymbol{A}, \boldsymbol{B}), 2$ $\mu \mathrm{m}(\boldsymbol{A}, \boldsymbol{D}, \mathbf{G}$; left panels), or $4 \mu \mathrm{m}$ ( $\mathbf{G}$; right panels).

continues to be expressed even in adult animals (P60; Fig. $1 F)$ consistent with recent studies (Barford et al., 2017).

Because of the punctate nature of NSG2 expression in dendrites, we determined whether NSG2 localized to synapses. In DIV14-DIV16 hippocampal neurons a portion of endogenous NSG2 (endo-NSG2) punctae was independent of both presynaptic Synapsin-1 (SYN1) and postsynaptic HOMER1 (Fig. 1G, arrow), whereas strong synaptic localization was observed for a subset of endo-NSG2 punctae (Fig. 1G, left panels, arrowheads). Quantification of colocalization showed that $\sim 30 \%$ of HOMER 1 punctae colocalized with NSG2. By comparison, NSG2 punctae were frequently found adjacent to, but not colocalized with SYN1 punctae (Fig. 1G, left arrowhead). In fact, some endo-NSG2 punctae were found colocalized with HOMER1 that were not adjacent to SYN1 punctae (Fig. 
A

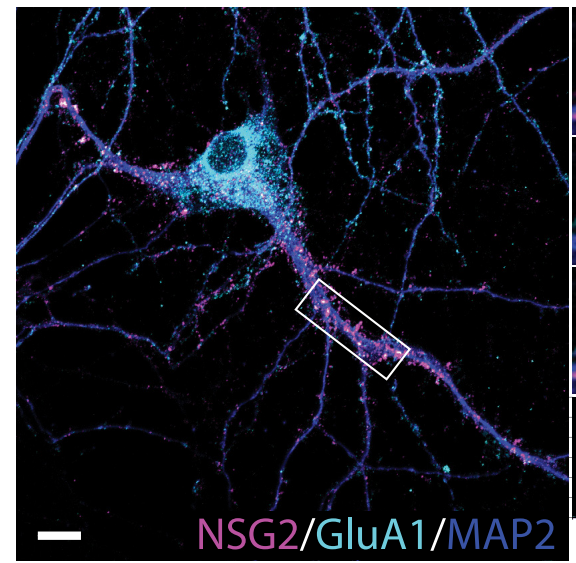

B

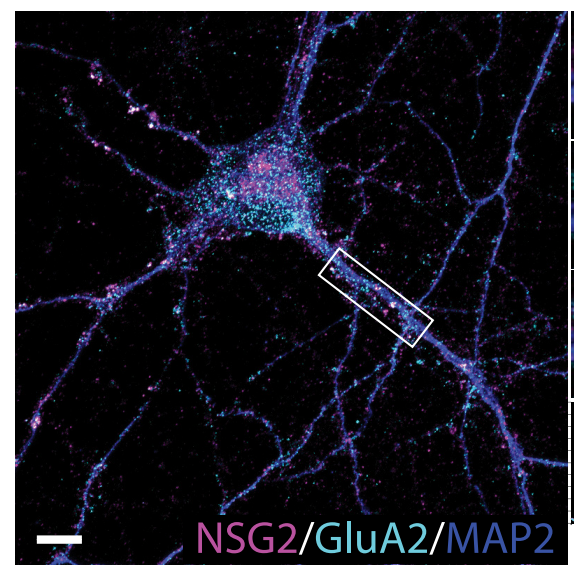

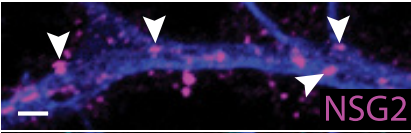
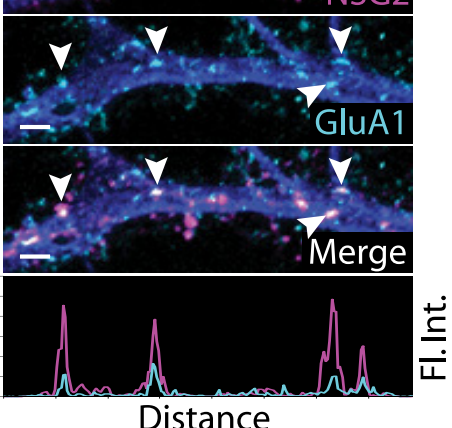

Distance

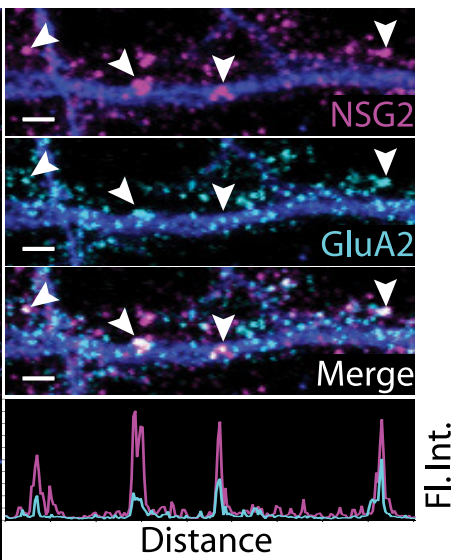

C

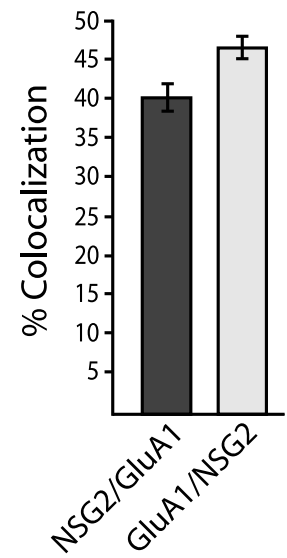

D

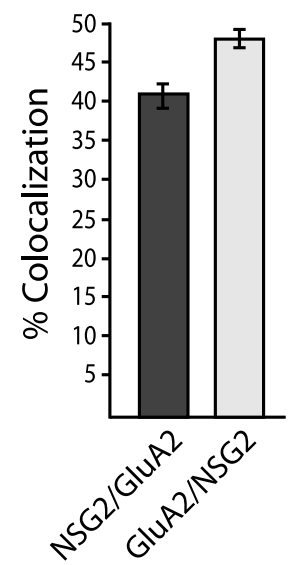

Figure 2. NSG2 colocalizes with AMPAR subunits GluA1 and GluA2. A, B, Representative confocal images of primary hippocampal neurons at DIV15 showing perinuclear and punctate NSG2 (magenta) in MAP2 ${ }^{+}$(blue) neurites along with surface expressed GluA1 punctae $(\boldsymbol{A}$, cyan), and GluA2 punctae ( $\boldsymbol{B}$, cyan). Separated color panels for individual markers from the boxed regions have been magnified for clarity (right panels). Lower right panels in $\boldsymbol{A}, \boldsymbol{B}$ show the colocalization profile for NSG2 (magenta)/GluA1 (cyan, panel $\boldsymbol{A})$ and NSG2 (magenta)/GluA2 (cyan, panel $\boldsymbol{B}$ ) across the indicated punctae (arrowheads). C, $\boldsymbol{D}$, Quantification of colocalization for NSG2 with surface GluA1 punctae (6423 NSG2 and 5392 GluA1 puncta from 10 neurons) and NSG2 with surface GluA2 punctae (6874 NSG2 and 5963 GluA2 puncta from nine neurons), respectively, from three independent cultures. Scale bars $=10 \mu \mathrm{m}(\boldsymbol{A}, \boldsymbol{B} ;$ left panels) and $2 \mu \mathrm{m}(\boldsymbol{A}, \boldsymbol{B}$; right panels).

$1 G$, left panels, far right arrowhead). Similar results were found with overexpressed NSG2-mC (Fig. 1G, right panels), indicating this construct behaves similarly to the endo-NSG2 protein. Interestingly, the percentage of HOMER1 punctae that colocalized with NSG2-mC was significantly increased compared to that for endo-NSG2 (Fig. 1H; endo-NSG2: $29.2 \pm 2.5 \%$; NSG2-mC: $37.0 \pm$ $2.0 \% ; * p=0.024)$. This happened despite the fact that the total densities of HOMER1 punctae for control and NSG2-mC were not significantly different (mCherry: 126 \pm 7 punctae/100 $\mu \mathrm{m}$; NSG2-mC: $133 \pm 10$ punctae/100 $\mu \mathrm{m} ; p=0.6)$. Thus, the increased colocalization between NSG2-mC and HOMER1 is likely a result of NSG2-mC entering HOMER $1^{+}$synapses that are not occupied by endo-NSG2. Taken together, these data suggest that NSG2 is present in a subset of excitatory synapses and is likely restricted to the PSD rather than presynaptic terminals. Furthermore, overexpressed NSG2-mC was not sufficient to drive formation of new $\mathrm{HOMER} 1^{+} \mathrm{PSD}$ but was able to enter new sites where endo-NSG2 was likely absent.

\section{NSG2 interacts with AMPAR subunits GluA1 and GluA2}

To determine whether NSG2 colocalizes with functional AMPAR-containing synapses, we used surface labeling with antibodies directed toward the extracellular N-termini of the GluA1 and GluA2 subunits. Figure $2 A, B$ shows representative confocal images of neurons (left panels) as well as individual neurites (right panels) from boxed regions. Results for both GluA1 and GluA2 revealed robust colocalization with individual NSG2 punctae (Fig. 2A, $B$, right panel arrowheads). Pooled data revealed that $\sim 40 \%$ of NSG2 punctae colocalized with either the GluA1 (Fig. $2 A, C$; NSG2/GluA1: $40.1 \pm 1.6 \%$ ) or GluA2 subunits (Fig. 2B,D; NSG2/GluA2: $41.0 \pm 1.5 \%$ ). Reciprocally, over $45 \%$ of GluA1 and GluA2 surface labeled punctae colocalized with NSG2, suggesting that nearly half of all AMPAR- 
containing PSDs contained NSG2 protein at DIV15 (Fig. 2C,D; GluA1/NSG2: $46.2 \pm 1.2 \%$; GluA2/NSG2: $48.0 \pm$ $0.9 \%)$. It is noteworthy that the percentage of colocalization between AMPAR subunits and NSG2 was slightly greater than that of HOMER1/NSG2, indicating that NSG2 is somewhat selective for AMPAR-containing PSDs. We additionally verified that overexpressed NSG2-mC colocalized with human GluA1 subunits in hPSNs at day 50 . While these data revealed significant colocalization of NSG2 with surface GluA1 in hPSNs (Extended Data Fig. 2-1), the inconsistent maturational state of individual hPSNs (Johnson et al., 2007) makes characterization of NSG2 function more difficult. Thus, all future experiments were conducted on cultured mouse hippocampal neurons.

We next determined whether NSG2 physically associates with AMPAR subunits GluA1 and GluA2. We first performed Co-IP from HEK293T cells co-expressing the fusion proteins NSG2-mC $(\sim 45 \mathrm{kDa})$ and either YFPGluA1 or GFP-GluA2 ( 128 and $125 \mathrm{kDa}$, respectively). Using an anti-mCherry monoclonal antibody we immunoprecipitated NSG2-mC and probed for YFP-GluA1 and GFP-GluA2 using antibodies specific for each subunit. Figure $3 A$ shows data from a capillary immunoelectrophoresis that demonstrate NSG2-mC was able to Co-IP both GluA1 and GluA2 independently (lanes marked IP: mCherry) when the two proteins were co-expressed in HEK293T cells. An IgG control antibody did not IP either NSG2 or AMPAR subunits demonstrating the specificity of the reaction (Fig. $3 A$; IP: $\lg G$ ); the heavy chain of the control mouse IgG antibody is detected by the antimouse secondary antibody used to detect GluA1 and GluA2. To further validate our findings, we used the traditional Co-IP/SDS-PAGE to probe for a NSG2-mC and GluA2 interaction (Extended Data Fig. 3-1, middle lane). In this case, 24-h incubation of lysates taken from HEK293T cells that expressed either protein separately showed significantly reduced Co-IP, suggesting that their coexpression in a cellular context may be necessary to drive NSG2-GluA2 interactions (Extended Data Fig. 3-1, right lane). We also performed the complementary experiment to verify the interaction of overexpressed proteins. Immunoprecipitation of either GluA1 or GluA2 using antibodies previously described (Schwenk et al., 2014) could Co-IP both overexpressed NSG2 (Fig. 3B, arrow) as well as endogenous NSG2 (Fig. 3C, arrow). In this case we used whole-cell lysates from HEK293T cells co-expressing either GFP-GluA2 or YFP-GluA1 with unlabeled NSG2 ( 19 $\mathrm{kDa}$ ) to demonstrate that non-specific interactions between the fluorophores played no role in the interactions. In Figure 3B, the lanes marked IP: GluA1 and IP: GluA2 indicate that both GluA1 and GluA2 were able to Co-IP NSG2 (lower band, arrow). Finally, we asked whether the interaction between NSG2 and GluA1 and GluA2 subunits of AMPARs occurs in vivo. Utilizing whole-brain protein lysates from $P 0$ mouse we found that immunoprecipitation of either GluA1 or GluA2 AMPAR subunits pulled down endo-NSG2 (Fig. 3C; lanes marked IP: GluA1 and IP: GluA2). Non-specific mouse lgG antibody did not pull down either AMPARs or NSG2 (Fig. 3A-C; lanes marked IP: IgG). Heavy and light chains of control IgG are present similar to heavy chain in Figure $3 A, B$; lowest bands in "IP: IgG" group are likely non-specific as they appear in all groups and are of lower molecular weight compared to NSG2.

\section{NSG2 expression affects AMPAR currents}

Since NSG2 colocalizes with and is found in a complex with AMPAR subunits GluA1 and GluA2, we next asked if manipulating NSG2 protein levels would alter AMPAR surface expression as well as excitatory synaptic transmission. Two CRISPR/Cas9 gRNAs were designed using the MIT CRISPR design tool to target the mouse NSG2 locus. A T7 endonuclease cleavage assay determined that both gRNA\#1 and gRNA\#2 created indels in the NSG2 locus in mouse Neuro-2a neuroblastoma cells. However, the efficiency (Extended Data Fig. 4-3C) of gRNA\#2 was significantly greater than gRNA\#1, so this one was used for all experiments in primary hippocampal neurons. Transduction of control CRISPR virus and NSG2 gRNA was performed at DIV4 and neurons were analyzed at DIV16-DIV18. Figure $4 A$, upper panels, shows robust NSG2 punctae (arrowheads) in neurons transduced with control CRISPR lentivirus. In contrast, neurons transduced with the CRISPR gRNA targeting NSG2 resulted in a complete knock-out of NSG2 (Fig. 4A, lower panels; NSG2 KO; arrow). Note that no effect on NSG2 expression was observed in adjacent, non-transduced neurons (Fig. 4A, lower panels, arrowhead). Interestingly, the quantification of NSG2 KO in neurons transduced with NSG2 CRISPR virus revealed that $\sim 70 \%$ of neurons $(n=$ $38 / 55$, from three independent cultures) had a complete $\mathrm{KO}$. To confirm specificity of CRISPR targeting, we analyzed NSG1 expression in neurons that expressed NSG2 gRNA and showed complete KO of NSG2 protein. These neurons showed no significant difference in the density of NSG1 punctae compared to neurons expressing control CRISPR constructs (Extended Data Fig. 4-3B; control: $346.3 \pm 137.75$ punctae/100 $\mu \mathrm{m}$; NSG2 KO: $354.3 \pm$ 134.26 punctae $/ 100 \mu \mathrm{m} ; p=0.97)$. To determine the effect of NSG2 KO on synaptic transmission we performed whole-cell patch clamp recordings on $\mathrm{GFP}^{+}$neurons that received the CRISPR-NSG2 KO virus. To eliminate possible contamination from the small proportion of neurons that did not show NSG2 KO, we used Lucifer yellow injections followed by post hoc analysis of KO efficiency by immunocytochemistry to correlate NSG2 levels with physiologic recording data (Extended Data Fig. 4-1; only cells with undetectable NSG2 signal were used for analysis). Figure $4 B$ shows representative traces of voltage-clamp recordings from neurons expressing control CRISPR (black) or NSG2 KO CRISPR virus (green). Compared to control neurons, NSG2 KO neurons showed a significant decrease in the frequency of mEPSCs, with no change in amplitude (Fig. 4B,C; frequency; control: $12.41 \pm 2.31 \mathrm{~Hz}$; NSG2 KO: $4.07 \pm 0.68 \mathrm{~Hz} ; * * p=0.001$; amplitude; control: $16.71 \pm 4.10$ pA; NSG2 KO: $15.84 \pm$ $4.47 \mathrm{pA} ; p=0.34)$. These effects were specific to synaptic currents as voltage-gated potassium currents remained unchanged (Extended Data Fig. 4-2). To probe the mechanism of how NSG2 might affect mEPSCs, we performed 

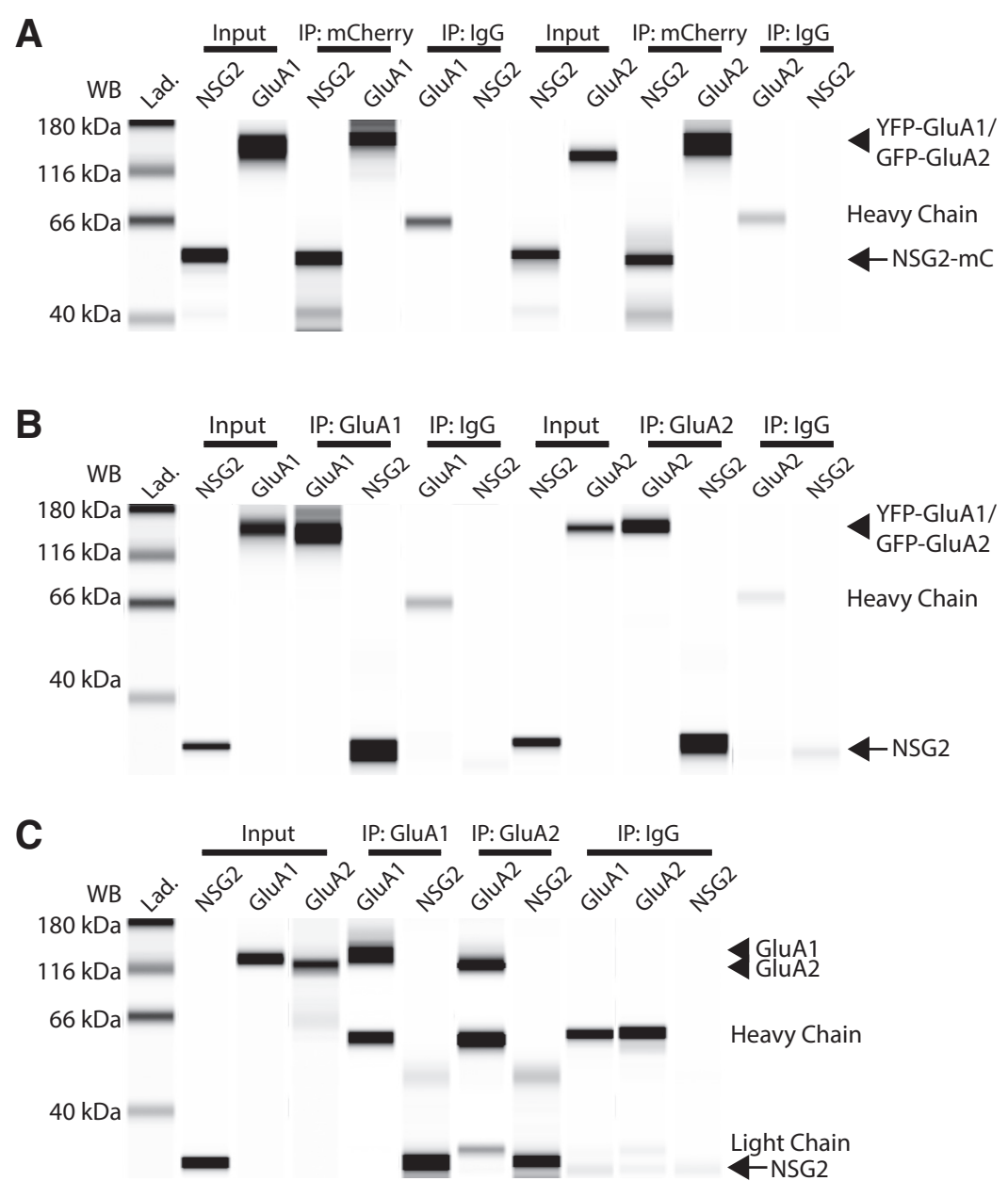

Figure 3. NSG2 interacts with AMPAR subunits GluA1 and GluA2. A, Co-IP of overexpressed, fluorophore-conjugated proteins. Representative digitized Western blottings from the automated Western blotting system (see Materials and Methods) showing that using the anti-RFP antibody to pull down NSG2-mC (IP: RFP, arrow) could Co-IP both YFP-GluA1 and GFP-GluA2 (IP: mCherry, arrowhead). A control IgG antibody did not IP nor detect any target proteins (IP: IgG); the heavy chain of mouse and anti-RFP was detected by the anti-mouse secondary antibody used to detect mouse anti-GluA1/2. NSG2-mC (arrow), YFP-GluA1, and GFP-GluA2 (arrowhead) were detected in the input (input). Traditional Western blotting techniques were performed to confirm that GluA2 co-immunoprecipitates with NSG2-mC (Extended Data Fig. 3-1). Samples were taken from HEK293T cells co-expressing NSG2-mC and either YFP-GluA1 or GFP-GluA2. B, Immunoprecipitation of overexpressed, unlabeled NSG2 with fluorophore-conjugated proteins with antibodies directed toward endogenous protein epitopes. Anti-GluA1 and anti-GluA2 antibodies immunoprecipitated YFP-GluA1 and GFP-GluA2, respectively (IP: GluA1 and IP: GluA2, arrowhead). Unlabeled NSG2 (arrow) coexpressed in HEK293T cells was also detected using these same conditions, confirming the Co-IP; control IgG did not IP nor detect any target proteins (IP: lgG). NSG2, YFP-GluA1, and GFP-GluA2 are detected in the input (input; GluA1; GluA2, arrowhead; NSG2, arrow). Lower bands in NSG2 lanes of control lgG pull down conditions is thought to be non-specific is found in all similar reaction conditions and migrates faster than NSG2. C, Immunoprecipitation from in vivo samples (whole-brain lysate of P0 mouse). Anti-GluA1 and anti-GluA2 antibodies immunoprecipitated endogenous GluA1 and GluA2 (IP: GluA1 and IP: GluA2, arrowhead) as well as endogenous NSG2 (arrow). All target proteins were detected in the input lanes (input; GluA1 and GluA2, arrowhead; NSG2, arrow). Control mouse IgG antibody did not pull down GluA1, GluA2, or NSG2 (IP: IgG). As expected, heavy chain and light chains from antibodies used for pull down were detected in most Co-IP conditions (all IP lanes).

immunocytochemical labeling of multiple synaptic proteins. NSG2 KO did not affect the density of presynaptic $\mathrm{SYN} 1^{+}$nor postsynaptic PSD95 ${ }^{+}$punctae along neurites (Fig. 4D; SYN1; control: $93 \pm 8$ punctae/100 $\mu \mathrm{m}$; NSG2 KO: $79 \pm 7$ punctae/100 $\mu \mathrm{m} ; p=0.46$; PSD95; control: $97 \pm 7$ punctae/ $100 \mu \mathrm{m}$; NSG2 KO: $80 \pm 9$ punctae/100 $\mu \mathrm{m} ; p=0.18)$. Interestingly, the fluorescence intensity of $\mathrm{PSD}^{+} 5^{+}$punctae was significantly decreased in NSG2 KO neurons compared to controls (Fig. $4 E$; control: $3.70 \pm$ 0.45 A.U.; NSG2 KO: $2.57 \pm 0.28$ A.U.; $* p=0.04)$. This occurred without a corresponding change in the density of GluA1- and GluA2-containing punctae along neurites (Fig. 4F; surface GluA $1^{+}$; control: $86 \pm 8$ punctae $/ 100 \mu \mathrm{m}$; NSG2 KO: $83 \pm 12$ punctae/100 $\mu \mathrm{m} ; p=0.86$ and surface GluA2 ${ }^{+}$; control: $94 \pm 7$ punctae/100 $\mu \mathrm{m}$; NSG2 KO: $74 \pm 12$ punctae/ $100 \mu \mathrm{m} ; p=0.18$ ). Together, these data indicate that the reduction in mEPSC frequency due to loss of NSG2 may be caused by a relatively subtle effect on postsynaptic scaffolding rather than a failure of AMPAR exocytosis. 
A

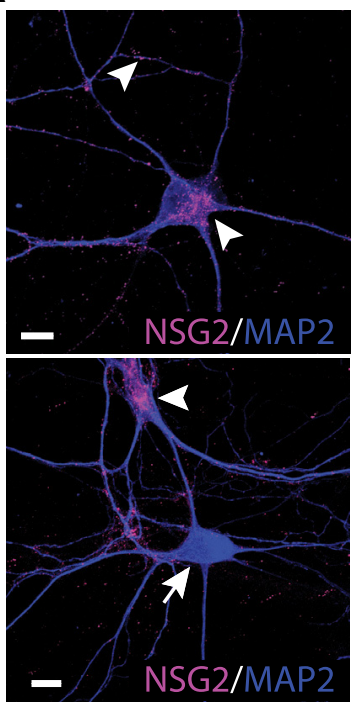

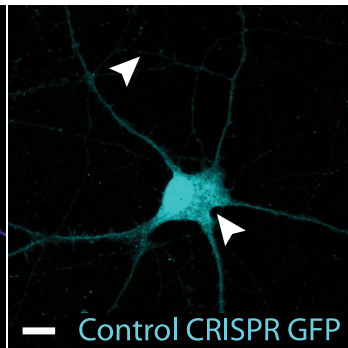
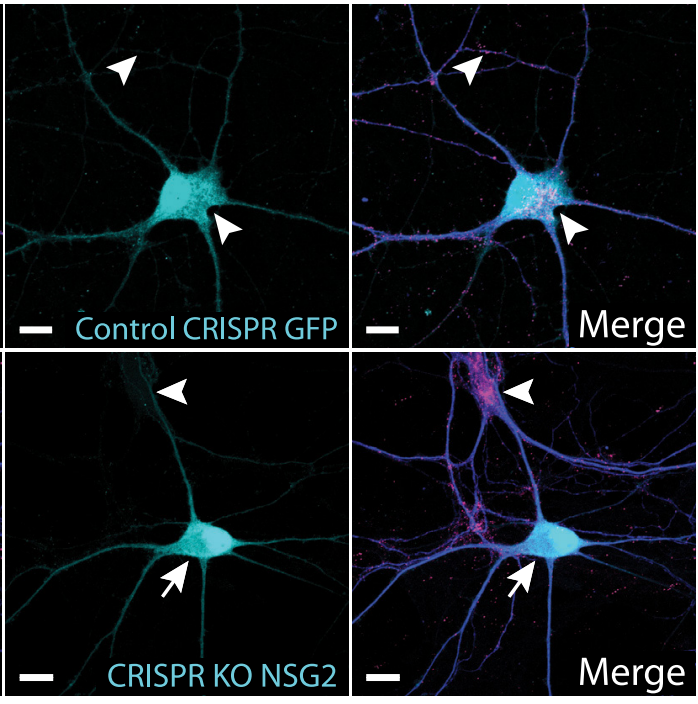

B

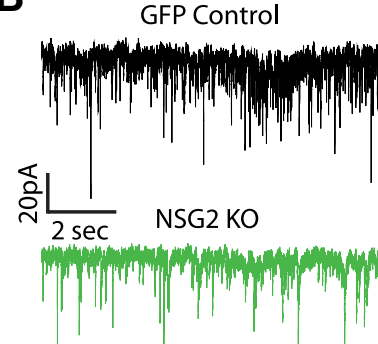

C

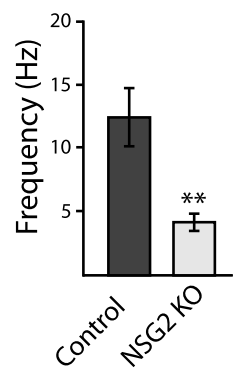

GFP Control

NSG2 KO
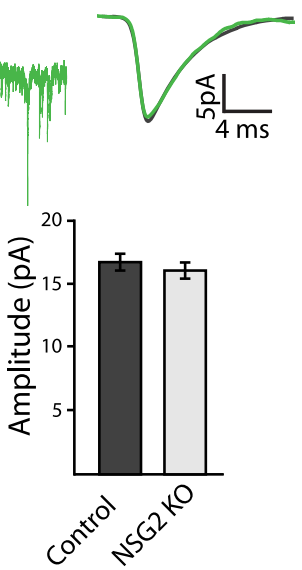
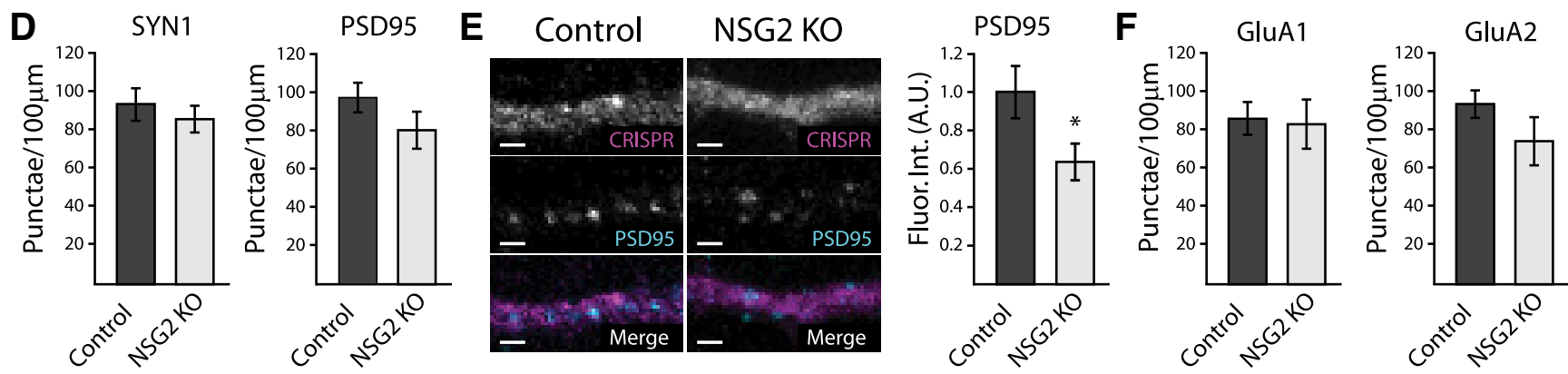

Figure 4. Knock-out of NSG2 decreases mEPSC frequency. $\boldsymbol{A}$, Representative confocal images of primary hippocampal neurons at DIV15 showing robust NSG2 (magenta) in MAP2 ${ }^{+}$(blue) neurons transduced with control CRISPR GFP lentivirus (cyan; top panels), whereas neurons transduced with CRISPR KO NSG2 lentivirus (cyan, bottom panels) show the absence of NSG2 (arrow; bottom panels); NSG2 (magenta) is present in an adjacent neuron not transduced with the CRISPR KO NSG2 lentivirus in the same field (arrowhead; bottom panels). B, Representative traces from whole-cell patch clamp recordings from neurons expressing either control CRISPR GFP (upper trace, black) or CRISPR KO NSG2 (bottom trace, green). Averaged mEPSCs from both control (black, $n=10)$ and NSG2 KO (green, $n=13$ ) are shown to the right. $\boldsymbol{C}$, Pooled data revealed a significant decrease in mEPSC frequency in neurons expressing NSG2 KO compared to cells expressing control CRISPR GFP ( $* p=0.001)$. The amplitude of mEPSCs was not significantly different between groups $(p=0.34)$. Data in the KO group were derived only from neurons devoid of NSG2 confirmed by post-recording immunostaining of Lucifer yellow injected neurons (Extended Data Fig. 4-1). NSG2 KO did not alter outward potassium I/V relationship (Extended Data Fig. 4-2). D, Quantification of presynaptic marker Synapsin1 ${ }^{+}$punctae $($control, $n=10$; NSG2 KO, $n=10 ; p=0.46$ ) and postsynaptic marker PSD95 ${ }^{+}$punctae (control, $n=9 ; \mathrm{NSG} 2 \mathrm{KO}, n=11 ; p=0.18$ ). $\boldsymbol{E}$, Representative confocal images illustrate PSD95 immunofluorescence (cyan) in neurons expressing CRISPR KO NSG2 (right panels) or controls CRISPR GFP (left panels). GFP expression from both groups (top panels) is presented in grayscale for clarity. Quantification revealed a significant reduction in PSD95 fluorescence intensity in neurons expressing NSG2 KO $(n=11)$ compared to controls $(n=9 ; * p=0.029)$. $\boldsymbol{F}$, Pooled data show that the number of surface GluA1 ${ }^{+}$punctae (left; control, $n=10$ and NSG2 KO, $n=9 ; p=0.86$ ) and surface GluA2 ${ }^{+}$punctae (right; control, $n=10$ and NSG2 KO, $n=9 ; p=0.18$ ) remained unchanged between groups. Bars represent mean \pm SEM. Scale bars $=10 \mu \mathrm{m}(\boldsymbol{A})$ and $1 \mu \mathrm{m}(\boldsymbol{E})$.

To test whether NSG2 can augment postsynaptic targeting of AMPARs, we used overexpression of NSG2-mC, which shows nearly identical synaptic targeting as endoNSG2 (Fig. 1D). Figure 5A shows representative traces of voltage-clamp recordings from neurons overexpressing mCherry (black) alone or NSG2-mC (red). Pooled data revealed that neurons expressing NSG2-mC showed a significant increase in mEPSC amplitude compared to those expressing mCherry alone (Fig. $5 A, B$; mCherry: $21.61 \pm 1.43 \mathrm{pA}$; NSG2-mC: $30.22 \pm 3.07 \mathrm{pA} ; * * p=$ $0.01)$. In contrast to NSG2 KO, no significant difference was observed for mEPSC frequency on NSG2 overexpression (Fig. $5 B$; mCherry: $12.88 \pm 2.91 \mathrm{~Hz}$; NSG-mC: $17.66 \pm 4.12 \mathrm{~Hz} ; p=0.35$ ). Immunocytochemical analyses revealed that the intensity of GluA1 and GluA2 staining showed a trend toward an increase but that did not reach significance (Fig. 5C,E; fluorescence intensity of GluA1; mCherry: $1.00 \pm 0.38$ A.U.; NSG2-mC: $1.63 \pm$ 0.54 A.U.; $p=0.36$ and GluA2; mCherry: $1.00 \pm 0.11$ A.U.; NSG2-mC: $1.32 \pm 0.21$ A.U.; $p=0.19$ ). Interestingly, while the number of surface GluA $1^{+}$punctae remained unchanged (Fig. 5C; mCherry: $92 \pm 10$ punctae/ 
A

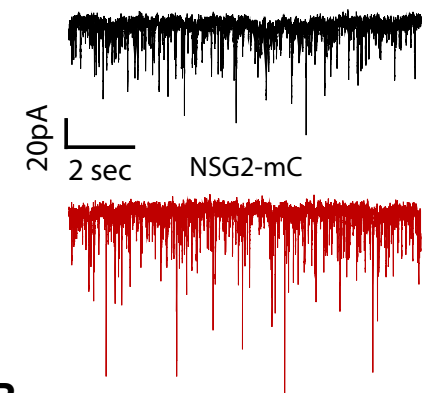

B

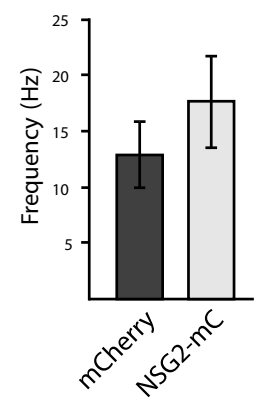

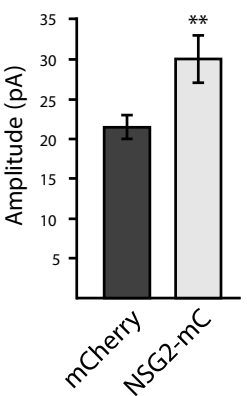

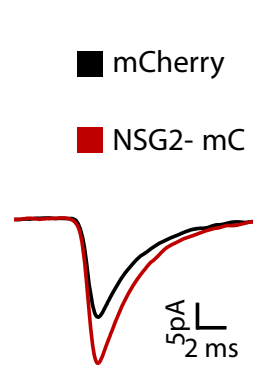

C
D

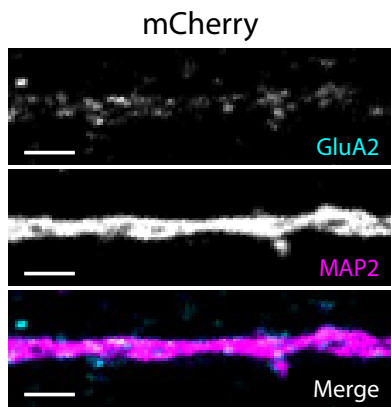

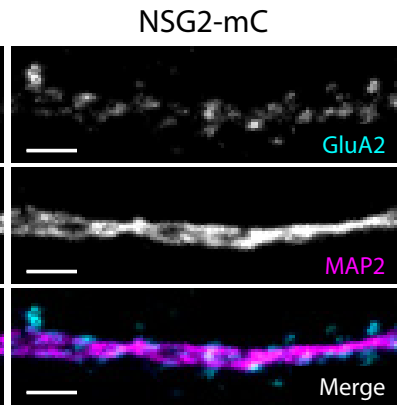

E
GluA2
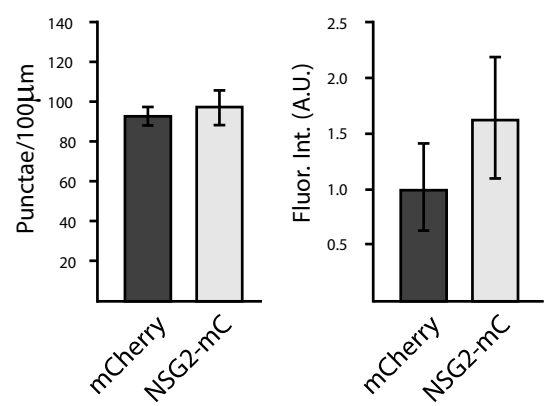

Figure 5. Overexpression of NSG2 increases mEPSC amplitude. $\boldsymbol{A}$, Representative traces from whole-cell patch clamp recordings from neurons expressing either mCherry alone (upper trace, black) or NSG2-mC (bottom trace, red). Averaged mEPSCs from both control (black, $n=11$ ) and NSG2-mC (red, $n=10$ ) are shown to the right. $\boldsymbol{B}$, Pooled data illustrate that neurons expressing NSG2-mC showed a significant increase in mEPSC amplitude compared to controls $(* * p=0.01)$, whereas the frequency of mEPSCs was not different between groups $(p=0.35)$. $C$, Neither GluA1+ punctae number $(p=0.58)$ nor relative fluorescence intensity $(p=0.36)$ were significantly different between groups (mCherry vs NSG2-mC). $\boldsymbol{D}$, Representative confocal images showing neurons expressing either mCherry alone (left panels) or NSG2-mC (right panels) stained for MAP2 (magenta) and surface GluA2 (cyan). Individual GluA2 and MAP2 expression from both groups (top two panels) are presented in grayscale for clarity. $\boldsymbol{E}$, Pooled data revealed the number of GluA2 ${ }^{+}$punctae was significantly increased in neurons expressing NSG2-mC $(n=10)$ compared to controls $(n$ $=10 ; * p=0.02$ ), while relative fluorescence tntensity remained unchanged $(p=0.19)$. Bars represent mean \pm SEM; A.U., arbitrary units. Scale bars $=2 \mu \mathrm{m}$.

$100 \mu \mathrm{m} ; \mathrm{NSG} 2-\mathrm{mC}: 97 \pm 8$ punctae/100 $\mu \mathrm{m} ; p=0.58)$, the number of $\mathrm{GluA}^{+}$punctae was significantly increased on NSG2-mC overexpression (Fig. 5E; mCherry: $94 \pm 6$ punctae/100 $\mu \mathrm{m}$; NSG2-mC: $123 \pm 10$ punctae/ $100 \mu \mathrm{m} ; * p=0.02)$. These data suggest that NSG2 is sufficient to drive increased surface expression of AMPARs into existing functional synapses, and potentially to a small proportion of previously silent synapses.

\section{Discussion}

While critical roles in endosomal trafficking and synaptic plasticity have been established for NSG1 and NSG3, no functional role for NSG2 has previously been demonstrated. Here, we present evidence that NSG2 is a novel modulator of excitatory synaptic function. We found NSG2 displays a punctate pattern of expression that is restricted from axons but found robustly throughout somatodendritic arbors. Approximately forty percentage of all PSDs that contained surface expressed AMPAR subunits also displayed colocalized NSG2 punctae. Reciprocal immunoprecipitation assays revealed that NSG2 interacts with both GluA1 and GluA2 AMPAR subunits in vitro and in vivo. CRISPR/Cas9-mediated knock-out of NSG2 in primary hippocampal neurons resulted in a significantly reduced frequency of AMPAR-mediated mEP$\mathrm{SCs}$, in the absence of a reduction in total surface expressed AMPAR levels. Surprisingly, overexpression of NSG2 caused a significant increase in the amplitude of AMPAR-mediated mEPSCs that was coincident with increased surface expression of the AMPAR subunit GluA2, but did not affect frequency as in the knock-out condition. Together, these data suggest NSG2 is a novel player in excitatory postsynaptic function potentially via trafficking of AMPAR and/or associated cargo proteins (see below).

While it remains unclear as to how NSG2 modulates excitatory synaptic function specifically, the preponderance of evidence points to the regulation of AMPAR surface expression via secretory trafficking. This is supported by our current findings in conjunction with a number of findings for other NSG family members. For instance, NSG3 facilitates clathrin-mediated endocytosis of AMPARs during agonist stimulated activity that causes synaptic depression (Xiao et al., 2006; Davidson et al., 2009). Overexpression of NSG3 was shown to cause internalization of GluA1 and GluA2, decreasing surface expression levels of AMPARs. Furthermore, chronic upregulation of NSG3 in a transgenic mice was shown to impair executive cognitive function (Xiao et al., 2006; Kruusmägi et al., 2007; Davidson et al., 2009; Vazdarjanova et al., 2011), likely via alterations in AMPAR trafficking and altered LTD like processes (Myers et al., 2006; 
Kim et al., 2007). In contrast, NSG1 has been shown to regulate the rate of AMPAR receptor recycling within the PSD (Steiner et al., 2005; Utvik et al., 2009). While no studies report the functional consequences of full-length NSG1 overexpression, inhibition of NSG1 function via a dominant negative peptide prevented constitutive recycling of GluA1/2-containing AMPARs following NMDAmediated internalization (Alberi et al., 2005; Steiner et al., 2005). Similarly, antisense-mediated suppression of NSG1 function severely impaired the amplitude of evoked potentials in organotypic hippocampal slices (Alberi et al., 2005). However, it is not clear whether the observed effect on amplitude was a consequence of a reduction in the number of AMPARs within individual synapses, or a reduction in the number of synapses that contained AMPARs. For example, Levy et al. (2015) demonstrate that the effect of changing levels of the MAGUK family of scaffolding proteins, specifically knock-down of PSD95 causes a reduction in AMPAR mEPSC frequency in dissociated hippocampal neurons by increasing the number of silent synapses, however without affecting mEPSC amplitudes (Levy et al., 2015). Thus, one explanation for our observations could be that in NSG2 KO neurons, a subset of synapses that would have contained NSG2 showed reduced or altered AMPAR localization that our methods were not sensitive enough to detect via immunocytochemistry. In this case, these synapses demonstrated increased failure rates or sub-threshold events which resulted in a decrease in mEPSC frequency. However, the absence of an effect on AMPAR surface expression suggests either subtle changes in AMPAR localization or an alternate mechanism. We cannot rule out a role of NSG2 on presynaptic function, although manipulation of NSG1 and NSG3 does not alter presynaptic release (Alberi et al., 2005; Davidson et al., 2009), and NSG2 is solely found in the PSD (Figs. 1, 2; Alberi et al., 2005; Steiner et al., 2005). While future studies are needed to elucidate the mechanistic details of how NSG2 is involved in regulating AMPAR trafficking, our data support a complementary role of NSG2 with that other NSG family members.

One mechanism likely involves secretion of AMPARs via post-Golgi endosomal vesicles that may contain individual or combinations of NSG family members depending on function. For instance, Yap and colleagues recently demonstrated that both NSG1 and NSG2 puncta in dendrites show approximately $60 \%$ overlapping expression with the remaining forty percentage not colocalized. Interestingly, a significant proportion of overlap was found in Rab $7^{+}$late endosomes traveling in a retrograde fashion which the authors conclude were likely destined for degradation. Smaller proportions of NSG1/2 puncta found in EEA $1^{+}$early endosomal, Rab $11^{+}$recycling endosomes, and Lamp $1^{+}$lysosomes (Yap et al., 2017). Most intriguingly, this analysis accounted for nearly all $\mathrm{NSG}^{+}$punctae, but $<80 \%$ of $\mathrm{NSG}^{+}$punctae. Thus, it remains to be determined whether there is an additional pool of unique, NSG2-containing vesicles. Evidence for divergent trafficking mechanisms stems from the fact that NSG1 was previously shown to be involved in a unique transcytosis mechanism for trafficking and sorting neuronal L1/Ng-
CAM to axons (Yap et al., 2008). More recently, Shi et al. (2018) have shown that NSG3 associates with motor proteins to coordinate dynamic microtubule dependent trafficking of LysoTracker ${ }^{+}$late endosome and lysosome related organelles within axons. Further evidence for NSG2's unique functions can be inferred by sequence homology and known NSG family protein-protein interactions. NSG1 has been shown to bind to L1/NgCAM, Stx13, GRIP1, and APP (Steiner et al., 2005; Yap et al., 2008; Norstrom et al., 2010), while NSG3 binds clathrin light chain (CLC), the AP1-3 adaptors, and PSD-95 (Xiao et al., 2006; Ha et al., 2012; Muthusamy et al., 2012). Due to the relatively low sequence homology between NSG family members (Muthusamy et al., 2009), it is likely that NSG2 has unique binding partners that can inform the non-overlapping functions of NSG2 and other NSG family members with respect to spatiotemporal divergence in their post-Golgi trafficking.

NSG2 was originally proposed to have a role in secretory trafficking for the following reasons: (1) it is a single-pass transmembrane protein enriched in the Golgi Apparatus and post-Golgi vesicular compartments, (2) its presence in the P3 subcellular fraction of whole-brain ultracentrifugation, and (3) it contains a Secretogranin III-like domain in its C terminus (Sabéran-Djoneidi et al., 1995). Recent evidence from Yap and colleagues confirmed the secretory capacity of NSG2. Blocking endocytosis using a dominant negative Rab5 resulted in accumulation of NSG2 on the surface of hippocampal neurons (Yap et al., 2017). Here, we found that surface GluA2 levels and consequently AMPAR mEPSC amplitude, were significantly increased on NSG2 overexpression, suggestive of a link between its role in the secretory pathway and surface AMPAR levels. It is especially intriguing that NSG2 has an asymmetric synaptic distribution, being present in approximately forty percentage of excitatory synapses. As mentioned, data from this and previous studies show that inhibiting either NSG1 or NSG2 significantly, but not completely, inhibits synaptic transmission. Thus, it will be critical to determine whether other NSG family members occupy the same, or divergent populations of synapses, and whether the effects of their inhibition are additive. Furthermore, understanding their temporal or activity dependence will be critical. For instance, do NSG family members mark specific subsets of synapses, or do NSG proteins transiently visit most or all synapses during development or during particular types of activity? Future investigations to answer these questions should reveal previously unappreciated functional differences between individual excitatory synapses across multiple brain regions.

\section{References}

Alberi S, Boda B, Steiner P, Nikonenko I, Hirling H, Muller D (2005) The endosomal protein NEEP21 regulates AMPA receptormediated synaptic transmission and plasticity in the hippocampus. Mol Cell Neurosci 29:313-319. CrossRef Medline

Barford K, Yap CC, Dwyer ND, Winckler B (2017) The related neuronal endosomal proteins NEEP21 (Nsg1) and P19 (Nsg2) have divergent expression profiles in vivo. J Comp Neurol 525:18611878. CrossRef Medline

Davidson HT, Xiao J, Dai R, Bergson C (2009) Calcyon is necessary for activity-dependent AMPA receptor internalization and LTD in 
CA1 neurons of hippocampus. Eur J Neurosci 29:42-54. CrossRef Medline

Dittgen T, Nimmerjahn A, Komai S, Licznerski P, Waters J, Margrie TW, Helmchen F, Denk W, Brecht M, Osten P (2004) Lentivirusbased genetic manipulations of cortical neurons and their optical and electrophysiological monitoring in vivo. Proc Natl Acad Sci USA 101:18206-18211. CrossRef Medline

Esteves da Silva M, Adrian M, Schätzle P, Lipka J, Watanabe T, Cho S, Futai K, Wierenga CJ, Kapitein LC, Hoogenraad CC (2015) Positioning of AMPA receptor-containing endosomes regulates synapse architecture. Cell Rep 13:933-943. CrossRef Medline

Floruta CM, Du R, Kang H, Stein JL, Weick JP (2017) Default Patterning Produces Pan-cortical Glutamatergic and CGE/LGElike GABAergic Neurons from Human Pluripotent Stem Cells. Stem Cell Reports 9:1463-1476. CrossRef Medline

Grabrucker A, Vaida B, Bockmann J, Boeckers TM (2009) Synaptogenesis of hippocampal neurons in primary cell culture. Cell Tissue Res 338:333. CrossRef Medline

Ha CM, Park D, Han JK, Jang JI, Park JY, Hwang EM, Seok H, Chang $S$ (2012) Calcyon forms a novel ternary complex with dopamine D1 receptor through PSD-95 protein and plays a role in dopamine receptor internalization. J Biol Chem 287:31813-31822. CrossRef Medline

Heckl D, Kowalczyk MS, Yudovich D, Belizaire R, Puram RV, McConkey ME, Thielke A, Aster JC, Regev A, Ebert BL (2014) Generation of mouse models of myeloid malignancy with combinatorial genetic lesions using CRISPR-Cas9 genome editing. Nat Biotechnol 32:941-946. CrossRef Medline

Johnson MA, Weick JP, Pearce RA, Zhang SC (2007) Functional neural development from human embryonic stem cells: accelerated synaptic activity via astrocyte coculture. J Neurosci 27:30693077. CrossRef Medline

Kang HJ, Kawasawa Yl, Cheng F, Zhu Y, Xu X, Li M, Sousa AM, Pletikos M, Meyer KA, Sedmak G, Guennel T, Shin Y, Johnson MB, Krsnik Z, Mayer S, Fertuzinhos S, Umlauf S, Lisgo SN, Vortmeyer A, Weinberger DR et al. (2011) Spatio-temporal transcriptome of the human brain. Nature 478:483-489. CrossRef Medline

Kim J, Lee S, Park K, Hong I, Song B, Son G, Park H, Kim WR, Park E, Choe HK, Kim H, Lee C, Sun W, Kim K, Shin KS, Choi S (2007) Amygdala depotentiation and fear extinction. Proc Natl Acad Sci USA 104:20955-20960. CrossRef Medline

Kruusmägi M, Zelenin S, Brismar H, Scott L (2007) Intracellular dynamics of calcyon, a neuron-specific vesicular protein. Neuroreport 18:1547-1551. CrossRef Medline

Lasiecka ZM, Yap CC, Katz J, Winckler B (2014) Maturational conversion of dendritic early endosomes and their roles in L1mediated axon growth. J Neurosci 34:14633-14643. CrossRef Medline

Levy JM, Chen X, Reese TS, Nicoll RA (2015) Synaptic consolidation normalizes AMPAR quantal size following MAGUK loss. Neuron 87:534-548. CrossRef Medline

Miller JA, Ding SL, Sunkin SM, Smith KA, Ng L, Szafer A, Ebbert A, Riley ZL, Royall JJ, Aiona K, Arnold JM, Bennet C, Bertagnolli D, Brouner K, Butler S, Caldejon S, Carey A, Cuhaciyan C, Dalley RA, Dee N (2014) Transcriptional landscape of the prenatal human brain. Nature 508:199-206. CrossRef Medline

Murillo JR, Goto-Silva L, Sánchez A, Nogueira FC, Domont GB, Junqueira M (2017) Quantitative proteomic analysis identifies proteins and pathways related to neuronal development in differentiated SH-SY5Y neuroblastoma cells. EuPA Open Proteom 16:1-11. CrossRef

Muthusamy N, Ahmed SA, Rana BK, Navarre S, Kozlowski DJ, Liberles DA, Bergson C (2009) Phylogenetic analysis of the NEEP21/calcyon/P19 family of endocytic proteins: evidence for functional evolution in the vertebrate CNS. J Mol Evol 69:319-332. CrossRef Medline
Muthusamy N, Faundez V, Bergson C (2012) Calcyon, a mammalian specific NEEP21 family member, interacts with adaptor protein complex 3 (AP-3) and regulates targeting of AP-3 cargoes. J Neurochem 123:60-72. CrossRef Medline

Muthusamy N, Chen YJ, Yin DM, Mei L, Bergson C (2015) Complementary roles of the neuron-enriched endosomal proteins NEEP21 and calcyon in neuronal vesicle trafficking. J Neurochem 132:2031. CrossRef Medline

Myers KM, Ressler KJ, Davis M (2006) Different mechanisms of fear extinction dependent on length of time since fear acquisition. Learn Mem 13:216-223. CrossRef Medline

Norstrom EM, Zhang C, Tanzi R, Sisodia SS (2010) Identification of NEEP21 as a ss-amyloid precursor protein-interacting protein in vivo that modulates amyloidogenic processing in vitro. $J$ Neurosci 30:15677-15685. CrossRef Medline

Sabéran-Djoneidi D, Marey-Semper I, Picart R, Studler JM, Tougard C, Glowinski J, Lévi-Strauss M (1995) A 19-kDa protein belonging to a new family is expressed in the Golgi apparatus of neural cells. J Biol Chem 270:1888-1893. Medline

Schwenk J, Baehrens D, Haupt A, Bildl W, Boudkkazi S, Roeper J, Fakler B, Schulte U (2014) Regional diversity and developmental dynamics of the AMPA-receptor proteome in the mammalian brain. Neuron 84:41-54. CrossRef Medline

Sharma K, Fong DK, Craig AM (2006) Postsynaptic protein mobility in dendritic spines: long-term regulation by synaptic NMDA receptor activation. Mol Cell Neurosci 31:702-712. CrossRef Medline

Shi L, Hines T, Bergson C, Smith D (2018) Coupling of microtubule motors with AP- 3 generated organelles in axons by NEEP21 family member calcyon. Mol Biol Cell 29:2055-2068. CrossRef Medline

Steiner P, Sarria J-CF, Glauser L, Magnin S, Catsicas S, Hirling H (2002) Modulation of receptor cycling by neuron-enriched endosomal protein of 21 kD. J Cell Biol 157:1197-1209. CrossRef Medline

Steiner P, Alberi S, Kulangara K, Yersin A, Sarria JC, Regulier E, Kasas S, Dietler G, Muller D, Catsicas S, Hirling H (2005) Interactions between NEEP21, GRIP1 and GluR2 regulate sorting and recycling of the glutamate receptor subunit GluR2. EMBO J 24: 2873-2884. CrossRef Medline

Utvik JK, Haglerød C, Mylonakou MN, Holen T, Kropf M, Hirling $H$, Skare O, Laake P, Ottersen OP, Haug FM, Davanger S (2009) Neuronal enriched endosomal protein of $21 \mathrm{kDa}$ colocalizes with glutamate receptor subunit GLUR2/3 at the postsynaptic membrane. Neuroscience 158:96-104. CrossRef Medline

Vazdarjanova A, Bunting K, Muthusamy N, Bergson C (2011) Calcyon upregulation in adolescence impairs response inhibition and working memory in adulthood. Mol Psychiatry 16:672-684. CrossRef Medline

Weick JP, Held DL, Bonadurer GF 3rd, Doers ME, Liu Y, Maguire C, Clark A, Knackert JA, Molinarolo K, Musser M, Yao L, Yin Y, Lu J, Zhang X, Zhang SC, Bhattacharyya A (2013) Deficits in human trisomy 21 iPSCs and neurons. Proc Natl Acad Sci USA 110:99629967. CrossRef Medline

Xiao J, Dai R, Negyessy L, Bergson C (2006) Calcyon, a novel partner of clathrin light chain, stimulates clathrin-mediated endocytosis. J Biol Chem 281:15182-15193. CrossRef Medline

Yap CC, Winckler B (2012) Harnessing the power of the endosome to regulate neural development. Neuron 74:440-451. CrossRef Medline

Yap CC, Wisco D, Kujala P, Lasiecka ZM, Cannon JT, Chang MC, Hirling H, Klumperman J, Winckler B (2008) The somatodendritic endosomal regulator NEEP21 facilitates axonal targeting of L1/ NgCAM. J Cell Biol 180:827-842. CrossRef Medline

Yap CC, Digilio L, McMahon L, Winckler B (2017) The endosomal neuronal proteins Nsg1/NEEP21 and Nsg2/P19 are itinerant, not resident proteins of dendritic endosomes. Sci Rep 7:10481. CrossRef Medline 